\title{
Supercritical Carbon Dioxide-Assisted Rapid Synthesis of Few-Layer Black Phosphorus for Hydrogen Peroxide Sensing
}

\author{
Shancheng Yan*a ${ }^{\mathrm{a}}$, Bojun Wang ${ }^{\mathrm{a}}$, Zhulan Wang ${ }^{\mathrm{b}}$, Dong Hu ${ }^{\mathrm{a}}, \mathrm{Xin} \mathrm{Xu}^{\mathrm{a}}$, Junzhuan Wang ${ }^{\mathrm{c}}$, Yi Shi*c \\ ${ }^{\mathrm{a}}$ School of Geography and Biological Information, Nanjing University of Posts and \\ Telecommunications, Nanjing 210023, P. R. China \\ ${ }^{\mathrm{b}}$ Community health service center of ZhongHuaMen, Nanjing 210046, China \\ ${ }^{\mathrm{c}}$ National Laboratory of Solid State Microstructures, School of Electronic Science and \\ Engineering, Nanjing University, Nanjing 210093, P. R. China
}

*Corresponding author. Tel.: +86-25 85866634; Fax: +86-25 85866634;

E-mail: yansc@njupt.edu.cn (Shancheng Yan)

yshi@nju.edu.cn (Yi Shi). 
Abstract: Solutions with large-scale dispersions of 2D black phosphorus (BP), often referred to as phosphorene, are obtained through solvent exfoliation. But, rapid phosphorene synthesis remains a challenge. Furthermore, although the chemical sensing capability of BP-based sensors has been theoretically predicted, its experimental verification remains lacking. In this study, we demonstrate the use of supercritical carbon dioxide-assisted rapid synthesis (5 h) of few-layer BP. In addition, we construct a non-enzymatic hydrogen peroxide $\left(\mathrm{H}_{2} \mathrm{O}_{2}\right)$ sensor based on few-layer BP for the first time to utilize BP degradation under ambient conditions. The proposed $\mathrm{H}_{2} \mathrm{O}_{2}$ sensor exhibits a considerably lower detection limit of $1 \times 10^{-7} \mathrm{M}$ compared with the general detection limit of $1 \times 10^{-7} \mathrm{M}$ to $5 \times 10^{-5} \mathrm{M}$ via electrochemical methods. Overall, the results of this study will not only expand the coverage of BP research but will also identify the important sensing characteristics of BP.

Keywords: Few-layered black phosphorus, Hydrogen peroxide sensing, Supercritical carbon dioxide; Rapid synthesis 


\section{Introduction}

The rediscovery of black phosphorus (BP) as a new single-element, 2D, layered material has recently aroused the interest of scientists from various fields (Abbas et al. 2015, Avsar et al. 2015, Ribeiro et al. 2015, Saito and Iwasa 2015). BP is a semiconductor that exhibits a direct, thickness-dependent band gap ranging from -0.3 $\mathrm{eV}$ in bulk $\mathrm{BP}$ to $-1.5 \mathrm{eV}$ in the monolayer (i.e., phosphorene) limit (Doganov et al. 2015, Wang Xiaomu et al. 2015b, Xiang et al. 2015). Two production methods for BP have been developed, namely, micromechanical exfoliation and liquid-phase exfoliation (Brent et al. 2014, Kang et al. 2015, Liu Han et al. 2015a, Yasaei et al. 2015). Although micromechanical exfoliation generally produces BP with the highest crystal quality, this method provides limited scalability. Alternatively, liquid-phase exfoliation via ultrasonication or shear mixing is a viable option for preparing phosphorene without intermediate chemical reactions. However, this approach requires a considerably long reaction time (over $12 \mathrm{~h}$ ) (Sreshtt et al. 2015, Woomer et al. 2015). Moreover, the degradation of BP under ambient conditions remains a serious consideration. The passivation and stability of BP has also been studied in many devices (Cheng et al. 2014, Kim Joon-Seok et al. 2015a, Liu Xiaolong et al. 2015d). However, these studies have mainly focused on the physical and transport properties of $\mathrm{BP}$, whereas minimal efforts have been exerted to expand the application of BP to other areas, such as in the chemical and biological fields. (Kou et al. 2014, Li et al. 2014, Li et al. 2015).

In this study, we demonstrated supercritical carbon dioxide $\left(\mathrm{CO}_{2}\right)$-assisted rapid 
synthesis of few-layer BP ( $5 \mathrm{~h}$; other studies required over $12 \mathrm{~h}$ ) for the first time. In addition, we constructed a non-enzymatic hydrogen peroxide $\left(\mathrm{H}_{2} \mathrm{O}_{2}\right)$ sensor based on few-layer $\mathrm{BP}$ to utilize $\mathrm{BP}$ degradation under ambient conditions. $\mathrm{H}_{2} \mathrm{O}_{2}$ is an important chemical that is widely used in the food, pharmaceutical, chemical, and biochemical industries. It is not only a by-product of several highly selective oxidases but is also an essential mediator in biology, medicine, industries, and many other fields. Therefore, accurate $\mathrm{H}_{2} \mathrm{O}_{2}$ determination has recently become extremely important (Kim Yura et al. 2015b, Koman et al. 2015, Liu Qingyun et al. 2015c, Zhang Jianxiu et al. 2015a). Our proposed $\mathrm{H}_{2} \mathrm{O}_{2}$ sensor exhibits a considerably lower detection limit of $1 \times 10^{-7} \mathrm{M}$ compared with the general detection limit of $1 \times 10^{-7}$ to $5 \times 10^{-5} \mathrm{M}$ via electrochemical methods. The results presented herein will not only expand the coverage of BP research but will also identify the important sensing characteristics of BP.

\section{Materials and methods}

\subsection{Materials and apparatus}

Black phosphorus crystals powder were purchased from a commercial supplier (Nanjing Longray Co., Ltd.) and stored in a dark $\mathrm{Ar}$ glovebox. N-monthylpyrrolidinone (NMP) were were obtained from Sigma Co., Ltd. Phosphate buffer (PBS) was prepared by mixing $\mathrm{Na}_{2} \mathrm{HPO}_{4}$ and $\mathrm{NaH}_{2} \mathrm{PO}_{4}$. All chemicals were used without further treatment. Ultrapure water used in the experiment was purified using the Millipore water purification system (Milli-Q Academic). Field emission 
scanning electron microscopy (FESEM) was used to determine the morphology of the samples on a Zeiss Ultra-plus spectrophotometer. Transmission electron microscopy (TEM) images were obtained using a JEOL model JEM 2100 instrument at an accelerating voltage of $200 \mathrm{kV}$. Raman spectra were recorded using a Renishaw Invia Reflex system combined with Peltiercooled charge-coupled device (CCD) detectors and a Leica microscope with an excitation wavelength of $514.5 \mathrm{~nm}$. AFM height images were taken on a Veeco Multimode 8. UV-vis absorption spectra (UV-3600) were measured by dispersing the sample in anhydrous ethanol at room temperature.

\subsection{Preparation of BP}

In a typical procedure, $10 \mathrm{mg}$ of $\mathrm{BP}$ powder was added into $20 \mathrm{~mL}$ of NMP sonicated in an ice-bath for $1 \mathrm{~h}$ at the power of $150 \mathrm{~W}$. Then, the mixture was transported to a preheated $\left(40{ }^{\circ} \mathrm{C}\right)$ stainless steel reactor, and $\mathrm{CO}_{2}$ was charged into the reactor to the desired pressure $(15 \mathrm{MPa})$. The mixture was reacted in the supercritical carbon dioxide for $3 \mathrm{~h}$. Subsequently, the products were cooled at room temperature, sonicated in an ice-bath for $1 \mathrm{~h}$, separated by centrifugation.

\subsection{Preparation of BP electrodes}

BP nanosheets were dissolved in the solution of anhydrous ethanol for more than $3 \mathrm{~min}$ at room temperature to give a suspension. The BP nanosheet electrode was prepared by casting $5 \mu \mathrm{L} \mathrm{BP}$ nanosheet solutions onto the pretreated glass carbon electrode (GCE) (effective area of $7 \mathrm{~mm}^{2}$ ) using micropipet and dried in the Ar 
glovebox.

\subsection{Electrochemical measurements}

The electrochemical measurements were carried out on an electrochemical analyzer CHI 760D (Shanghai Chenhua Instrument Company, Shanghai) in a conventional three-electrode configuration with a saturated calomel electrode (SCE) as the reference electrode, with a Pt foil as the counter electrode, and the modified BP nanosheet electrode as the working electrode. The CVs were in $40 \mathrm{~mL}$ of 0.2 $\mathrm{mmol} \cdot \mathrm{L}^{-1} \quad \mathrm{~N}_{2}$-saturated PBS (pH 7.0). All electrochemical experiments were performed at room temperature, $25 \pm 2{ }^{\circ} \mathrm{C}$.

\section{Results and discussion}
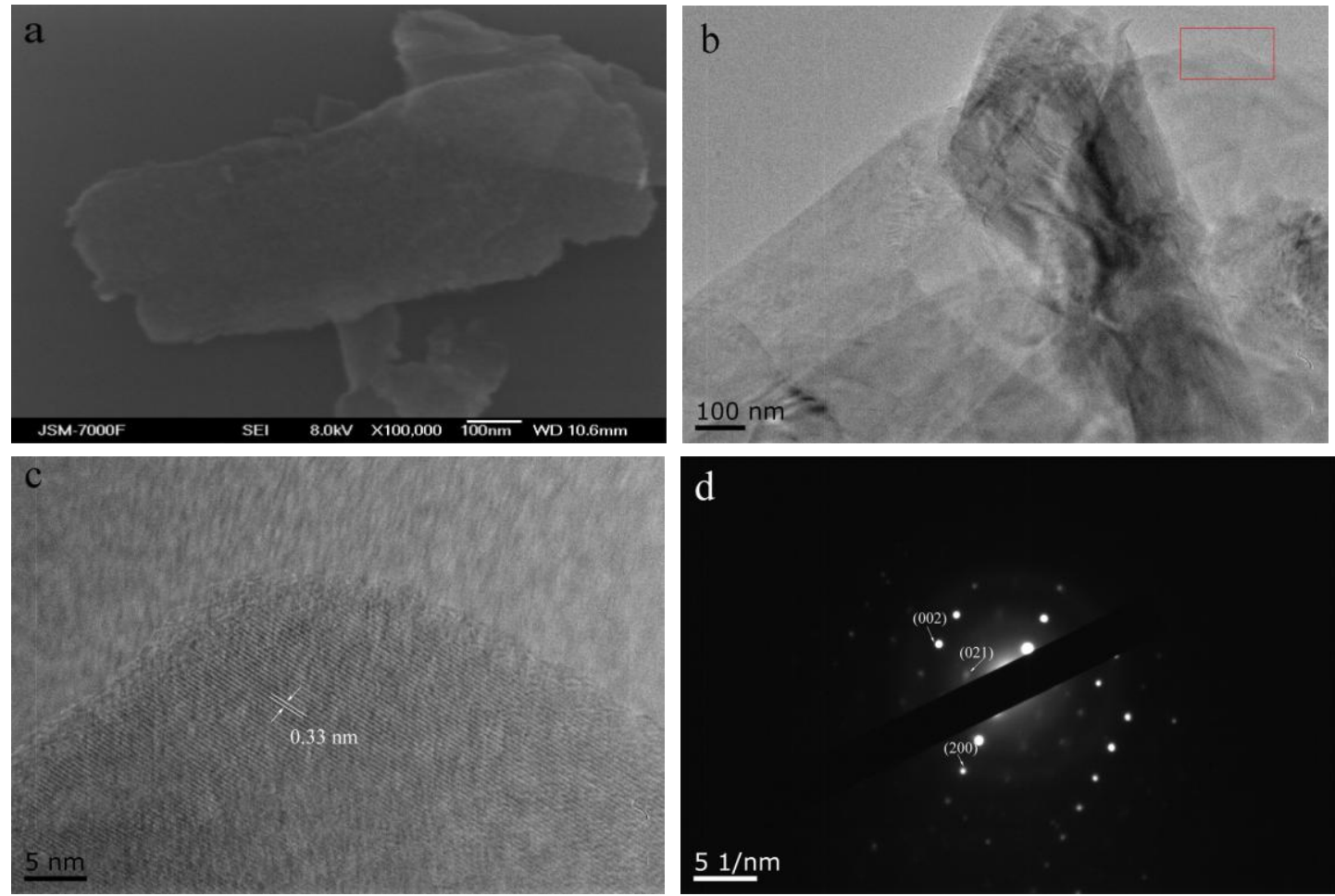

Figure 1 Morphology characterization of the few-layered black phosphorus. (a) 
FESEM image of few-layered black phosphorus. (b) Its TEM image. (c) HRTEM image of the area marked a red rectangular in (b). (d) SAED pattern of the black phosphorus nanosheet sample.

Fig. 1a shows the typical field-electron scanning electron microscopy image of the few-layer $\mathrm{BP}$ nanosheet sample. The $\mathrm{BP}$ nanosheet samples on the $\mathrm{SiO}_{2} / \mathrm{Si}$ substrate are transparent (Fig. 1a), which indicate that the BP samples are considerably thin. Supporting information Figure 1c and 1d show the photographs of the raw bulk BP and the BP treated with sonication and supercritical $\mathrm{CO}_{2}$. Figs. $1 \mathrm{~b}$ and 1c respectively show the low- and high-resolution transmission electron microscopy (HRTEM) images of the few-layer BP nanosheet sample prepared using the supercritical $\mathrm{CO}_{2}$-assisted route from the bulk $\mathrm{BP}$ crystal transferred directly onto the ultrathin carbon transmission electron microscope (TEM) grid. The typical HRTEM image shows the high crystalline quality of the as-transferred BP nanosheet sample onto the TEM grid. Moreover, the HRTEM image of the BP nanosheet obtained from the red rectangular area in Fig. $1 \mathrm{~b}$ shows lattice fringes of $0.33 \mathrm{~nm}$ (Fig. 1c), which can be ascribed to the (021) plane of the BP crystal (Late 2015). Overall, the results of the HRTEM analysis provide strong evidence that BP nanosheets exfoliated using supercritical $\mathrm{CO}_{2}$ maintain their crystalline nature. Fig. $2 \mathrm{~d}$ shows the typical selected area electron diffraction pattern of the few-layer BP samples, which confirms the highly 2D nature of the nanosheet. Further evidence of the chemical nature and purity of the supercritical $\mathrm{CO}_{2}$-assisted exfoliated flakes is provided via energy-dispersive X-ray analysis (EDXA). The EDXA result of the BP nanosheet is 
shown in Supporting Information Fig. 2. In the EDXA spectrum, the peaks of phosphorus are pronounced and no other peak is found except for those of copper and carbon, which originate from the copper microgrid with amorphous carbon used to support the sample. This result indicates that the product is phosphorus. The EDXA spectrum also confirms that the only elements (other than phosphorus) detected at significant levels are those associated with the TEM grid, which suggests that the produced nanosheet exhibits extremely high purity that is consistent with its crystalline nature.
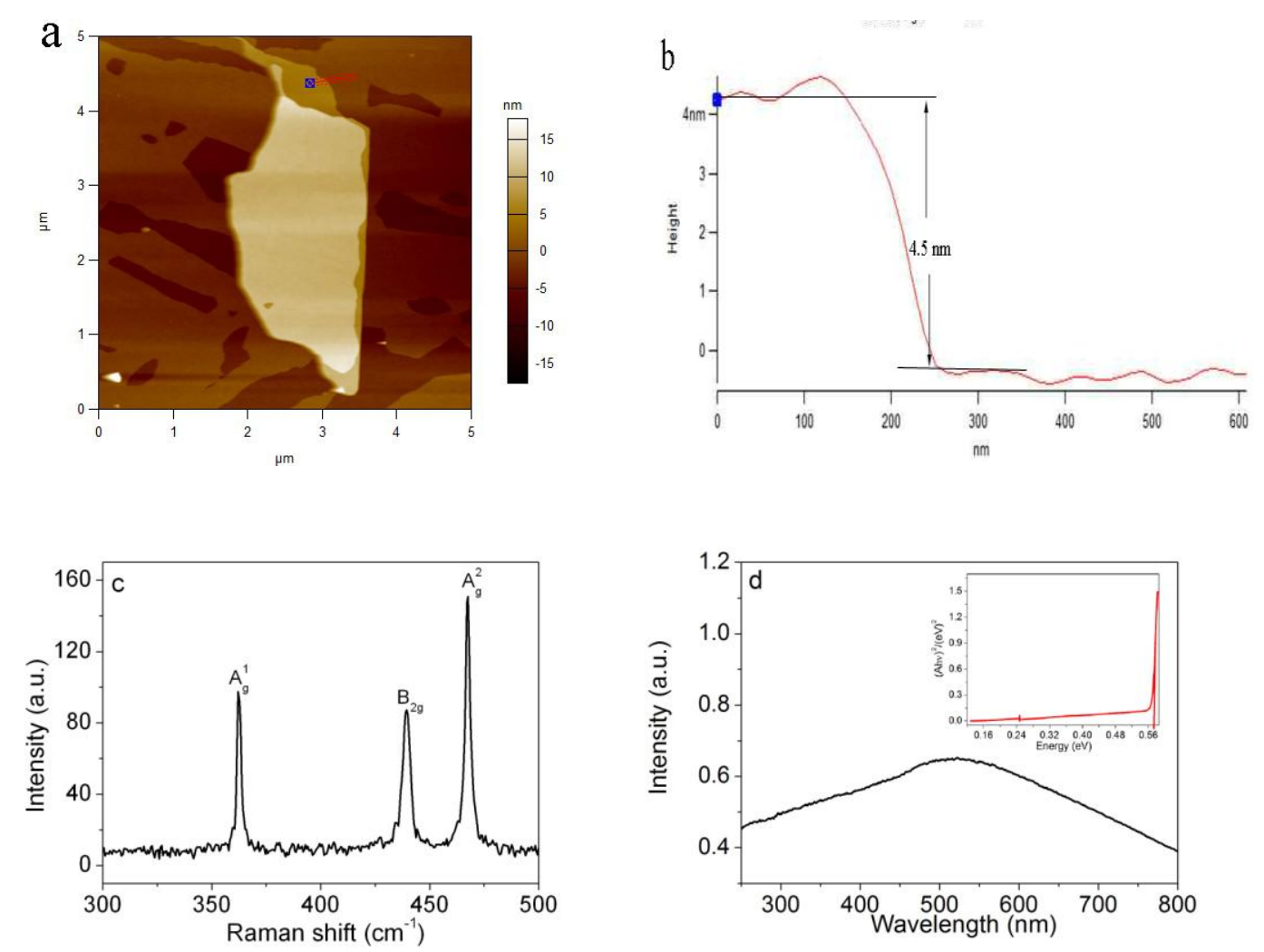

Figure 2 (a) Typical AFM image of the few-layered black phosphorus nanosheet sample deposited on $100 \mathrm{~nm} \mathrm{SiO}_{2} / \mathrm{Si}$ substrate and (b) the corresponding AFM height profile of the sample. (c) Raman spectrum of the few-layered black phosphorus. (d) UV/Vis absorption spectrum of the few-layered black phosphorus in anhydrous 
ethanol (Inset is band gap calculation).

The thickness values of the BP nanosheet samples are further determined via atomic force microscopy (AFM). Figs. 2a and 2b present the typical AFM image and height profile, which confirms that the height of the nanosheet is $\sim 4.5 \mathrm{~nm}$. This finding indicates the few-layer nature of the BP nanosheet sample. Previous AFM measurements have suggested that a single-layer phosphorene is $\sim 0.9 \mathrm{~nm}$ thick (Liu Han et al. 2014). Therefore, our AFM results suggest that large nanosheets predominantly consist of three to five phosphorene layers. We have also found single-layer and bilayer BP in the product. In addition, the AFM result of the supercritical $\mathrm{CO}_{2}$-assisted exfoliated phosphorene on $\mathrm{Si} / \mathrm{SiO}_{2}$ substrates shows the presence of various shapes and sizes of phosphorene, with flakes as large as $\sim 4 \mu \mathrm{m} \times$ $10 \mu \mathrm{m}$ (Supporting Information Fig. 5). Fig. 2c shows the typical Raman spectrum of the few-layer BP samples recorded at room temperature using $514.5 \mathrm{~nm}$ laser. Three prominent peaks can be ascribed to an out-of-plane phonon mode $\mathrm{A}_{\mathrm{g}}{ }^{1}$ at $362.2 \mathrm{~cm}^{-1}$ and to two in-plane modes, namely, $\mathrm{B}_{2 \mathrm{~g}}$ and $\mathrm{A}_{\mathrm{g}}{ }^{2}$, at $439.5 \mathrm{~cm}^{-1}$ and $467.6 \mathrm{~cm}^{-1}$, respectively. Compared with that of the bulk $\mathrm{BP}$, the $\mathrm{B}_{2 \mathrm{~g}}$ and $\mathrm{A}_{\mathrm{g}}{ }^{2}$ modes of the $\mathrm{BP}$ nanosheet are both red-shifted by $\sim 10 \mathrm{~cm}^{-1}$, whereas the $\mathrm{A}_{\mathrm{g}}{ }^{1}$ mode is blue-shifted by only $5 \mathrm{~cm}^{-1}$ (Fig. 2c). Compared with that of the bulk BP, the Raman spectrum of the BP nanosheet is slightly shifted toward higher wavenumbers, which is ascribed to the ultrathin nanosheets, as reported in previous studies (Wang Hui et al. 2015a). Furthermore, ultraviolet-visible (UV/Vis) absorption spectroscopy was performed to investigate the optical property of the produced BP nanosheet. The optical absorption 
spectra of BP nanosheets display a broad absorption band (which peaks at $\sim 520 \mathrm{~nm}$ ) in the UV/Vis spectra (Sun et al. 2015, Zhang Xiao et al. 2015b). The band gap of the as-prepared $\mathrm{BP}$ is estimated from the UV/Vis absorption spectra to be $0.57 \mathrm{eV}$ (inset in Fig. 2d), which is larger than that of the bulk BP.
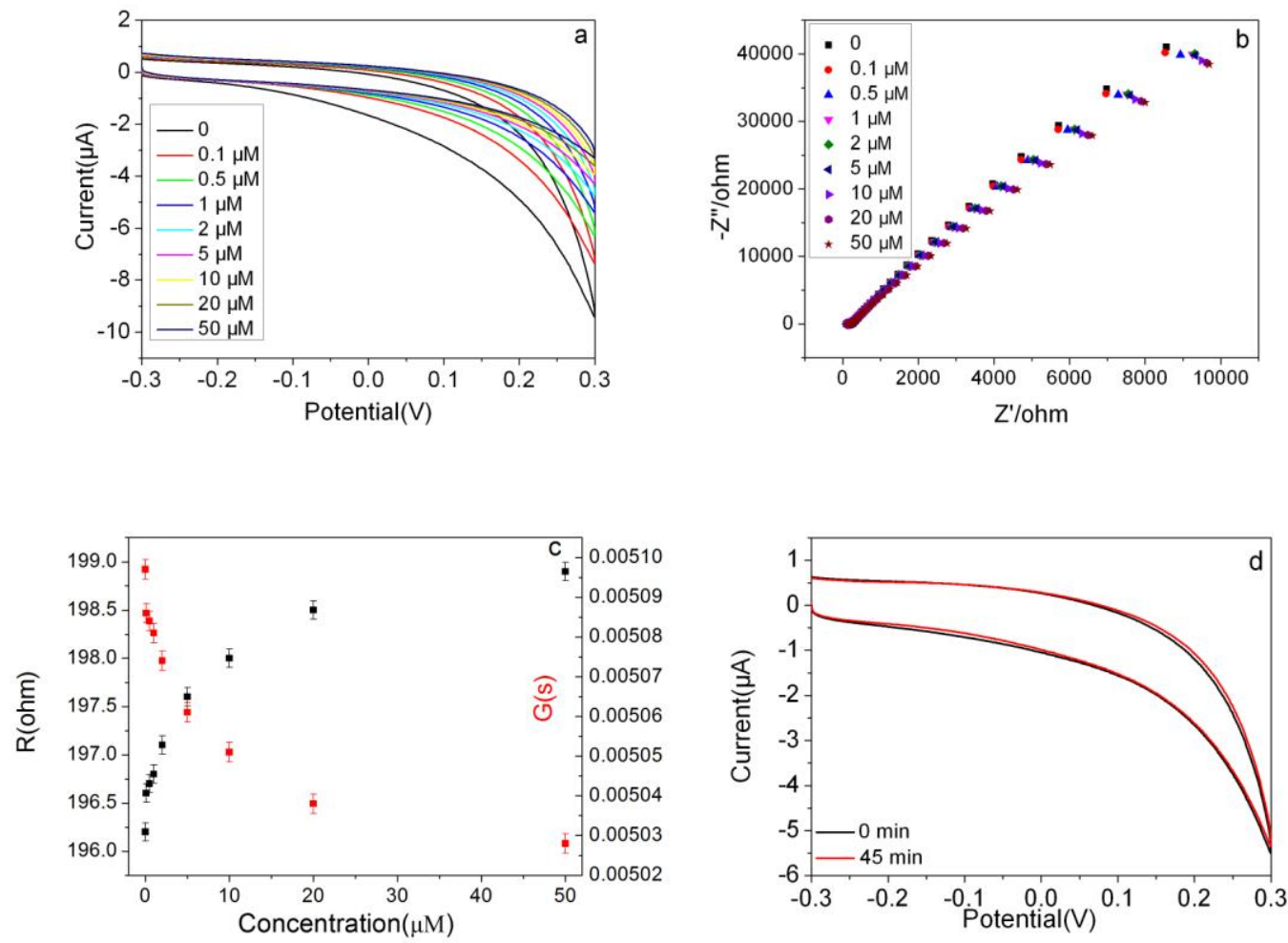

Figure 3 (a) Cyclic voltammograms of the few-layered black phosphorus nanosheet sample in $\mathrm{N}_{2}$-saturated PBS containing $\mathrm{H}_{2} \mathrm{O}_{2}$ at concentrations of $0,0.1,0.5,1,2,5$, 10, 20, and $50 \mu \mathrm{M}$; (b) EIS of the few-layered black phosphorus nanosheet sample in $\mathrm{N}_{2}$-saturated PBS. The frequency range was from $1 \mathrm{~Hz}$ to $100 \mathrm{kHz}$. (c) Relationships between the electrochemical impedance, electrochemical conductance and different concentrations of $\mathrm{H}_{2} \mathrm{O}_{2}$. (d) Cyclic voltammograms of the few-layered black phosphorus nanosheet sample in $\mathrm{N}_{2}$-saturated PBS with different time.

Although the accomplishments of BP appear promising, BP degradation under 
ambient conditions because of the oxidation of phosphorus remains a serious consideration. Therefore, researchers have investigated the environmental instability of few-layer BP under ambient conditions (Island et al. 2015). Some BP devices are encapsulated with hexagonal boron nitride in a layer-by-layer manner under an inert argon environment with minimized water and oxygen content (Avsar et al. 2015). Accordingly, to utilize BP degradation under ambient conditions, we constructed a non-enzymatic $\mathrm{H}_{2} \mathrm{O}_{2}$ sensor based on few-layer $\mathrm{BP}$ for the first time using the electrochemical impedance test. To evaluate the potential of the BP nanosheet in electrochemical detection, we investigated its performance via chronoamperometry. Fig. 3a shows the cyclic voltammetry (CV) data recorded in a solution of $\mathrm{N}_{2}$-saturated phosphate buffered saline (PBS). Each successive injection of $\mathrm{H}_{2} \mathrm{O}_{2}$ resulted in a decrease in $\mathrm{CV}$ area, which indicated the total decrease in charges. The charges were calculated from the cyclic voltammograms according to the following equation:

$$
\mathrm{C}=\left(\mathrm{q}_{\mathrm{a}}+\mathrm{q}_{\mathrm{b}}\right) / 2 / \triangle \mathrm{V}
$$

Where $\mathrm{q}_{\mathrm{a}}$ and $\mathrm{q}_{\mathrm{b}}$ are the sum of the anodic and cathodic voltammetric charges in the anodic and cathodic scan, respectively; and $\triangle \mathrm{V}$ is the potential range of the cyclic voltammogram. The increase in BP impedance is caused by the oxidation of BP. Therefore, current decreases with the addition of $\mathrm{H}_{2} \mathrm{O}_{2}$. Electrochemical impedance spectroscopy is a widely used tool in studying electron transfer between an electrolyte and an electrode surface. Fig. $3 \mathrm{~b}$ shows the impedance plots of the BP nanosheet at frequencies ranging from $1 \mathrm{~Hz}$ to $100 \mathrm{KHz}$. Fig. 3c shows the calibration curve among electrochemical impedance, electrochemical conductance, and different $\mathrm{H}_{2} \mathrm{O}_{2}$ 
concentrations. The response of electrochemical impedance increased with the increase in $\mathrm{H}_{2} \mathrm{O}_{2}$ concentration ranging from $1 \times 10^{-7} \mathrm{M}$ to $5 \times 10^{-5} \mathrm{M}$, with a considerably low detection limit of $1 \times 10^{-7} \mathrm{M}$, which was consistent with the aforementioned results (Fig. 3a). The response of the BP nanosheet electrode is considerably better than that of other sensor electrodes based on graphene or other 2D layered materials (Liu Jian et al. 2015b). The reproducibility of the proposed $\mathrm{H}_{2} \mathrm{O}_{2}$ sensors for BP nanosheet electrode was investigated by replicating measurements of five BP nanosheet electrodes. The variation coefficient of five measurements was 4.8\%. Compared with glass carbon electrode (Supporting Information Fig. 6), the changes in the BP nanosheet electrode are evident. Moreover, the degradation of the electrodes is apparently severe and can be attributed to high $\mathrm{H}_{2} \mathrm{O}_{2}$ concentrations because the $\mathrm{BP}$ nanosheet can be oxidized by $\mathrm{H}_{2} \mathrm{O}_{2}$, which may increase the electrochemical impedance of the electrodes. The curves of the differential pulse voltammetry are also consistent with this result (Supporting Information Fig. 7). The cyclic voltammograms of the few-layer BP nanosheet electrode in $\mathrm{N}_{2}$-saturated PBS at different durations are shown in Fig. $3 \mathrm{~d}$ to demonstrate the stability of the BP nanosheet electrode. The original electrochemical characteristic of the BP nanosheet electrode was maintained after 45 min of being in the $\mathrm{N}_{2}$-saturated PBS. This result suggests that our electrochemical detection results are credible despite the changes we make in the amount of $\mathrm{H}_{2} \mathrm{O}_{2}$. Furthermore, we investigated the stability of the BP nanosheet electrode in a certain amount of $\mathrm{H}_{2} \mathrm{O}_{2}(2 \mu \mathrm{M})$ (Supporting Information Fig. 8) and found that the BP nanosheet electrode was stable in the presence of $\mathrm{H}_{2} \mathrm{O}_{2}$ for a 
certain period.

\section{Conclusions}

A controllable supercritical $\mathrm{CO}_{2}$-assisted method for the rapid $(5 \mathrm{~h})$ synthesis of BP nanosheets was described for the first time, and the chemical sensing capability of a BP nanosheet as an $\mathrm{H}_{2} \mathrm{O}_{2}$ sensor was assessed. To utilize BP degradation under ambient conditions, we constructed a non-enzymatic $\mathrm{H}_{2} \mathrm{O}_{2}$ sensor based on few-layer BP for the first time. The proposed $\mathrm{H}_{2} \mathrm{O}_{2}$ sensor exhibited a considerably lower detection limit of $1 \times 10^{-7} \mathrm{M}$ compared with the general detection limit of $1 \times 10^{-7} \mathrm{M}$ to $5 \times 10^{-5} \mathrm{M}$ via electrochemical methods. We believe that our findings can provide a new insight into the extensively studied BP.

Acknowledgements: This work was financially supported by the National Basic Research Program of China (973 Program: 2013CB932903), the National Science Foundations of China (No. 61205057, No. 11574136), Qing Lan Project, the '1311 Talent Plan' Foundation of Nanjing University of Posts and Telecommunications, Six talent peaks project in Jiangsu Province (JY-014), the Open Research Fund of State Key Laboratory of Bioelectronics, Southeast University, and Jiangsu Provincial Key R \& D Program (Grant No. BE2015700).

\section{References}

Abbas, A. N., Liu, B., Chen, L., Ma, Y., Cong, S,, Aroonyadet, N., Koepf, M., Nilges, T., Zhou, C., 2015. ACS Nano 9, 5618-5624.

Avsar, A., Vera-Marun, I. J., Tan, J. Y., Watanabe, K., Taniguchi, T., Castro-Neto, A. H., Oezyilmaz, B., 2015. ACS Nano 9, 4138-4145.

Brent, J. R., Savjani, N., Lewis, E. A., Haigh, S. J., Lewis, D. J., O'Brien, P., 2014. Chem. Commun. 50, 13338-13341. 
Cheng, R., Li, D., Zhou, H., Wang, C., Yin, A., Jiang, S., Liu, Y., Chen, Y., Huang, Y., Duan, X., 2014. Nano Lett. 14, 5590-5597.

Doganov, R. A., O'Farrell, E. C. T., Koenig, S. P., Yeo, Y. T., Ziletti, A., Carvalho, A., Campbell, D. K., Coker, D. F., Watanabe, K., Taniguchi, T., 2015. Nature Comm. 6, 6647.

Island, J. O., Steele, G. A., Van der Zant, H. S. J., Castellanos-Gomez, A., 2015. Environmental instability of few-layer black phosphorus. 2D Materials 2, 011002.

Kang, J., Wood, J. D., Wells, S. A., Lee, J. H., Liu, X., Chen, K. S., Hersam, M. C., 2015. ACS Nano 9, 3596-3604.

Kim, J. S., Liu, Y., Zhu, W., Kim, S., Wu, D., Tao, L., Dodabalapur, A., Lai, K., Akinwande, D., 2015. Sci. Rep. 5, 8989.

Kim, Y., Park, J. Y., Kim, H. Y., Lee, M., Yi, J., Choi, I., 2015. Chem. Commun. 51, 15370-15373.

Koman, V. B., Santschi, C., Von Moos, N. R., Slaveykova, V. I., Martin, O. J. F., 2015. Biosens. Bioelectron. 68, 245-252.

Kou, L., Frauenheim, T., Chen, C., 2014. J. Phys. Chem. Lett. 5, 2675-2681.

Late, D. J., 2015. ACS Appl. Mater. Interfaces 7, 5857-5862.

Li, L., Yu, Y., Ye, G. J., Ge, Q., Ou, X., Wu, H., Feng, D., Chen, X. H., Zhang, Y., 2014. Nature Nanotech. 9, 372-377.

Li, L. K., Ye, G. J., Tran, V., Fei, R. X., Chen, G. R., Wang, H. C., Wang, J., Watanabe, K., Taniguchi, T., Yang, L., Chen, X. H., Zhang, Y. B., 2015. Nature Nanotech. 10, 608-614.

Liu, H., Du, Y., Deng, Y., Ye, P. D., 2015. Chem. Soc. Rev. 44, 2732-2743.

Liu, H., Neal, A. T., Zhu, Z., Luo, Z., Xu, X., Tomanek, D., Ye, P. D., 2014. ACS Nano 8, 4033-4041.

Liu, J., Bo, X., Zhao, Z., Guo, L., 2015. Biosens. Bioelectron. 74, 71-77.

Liu, Q., Yang, Y., Li, H., Zhu, R., Shao, Q., Yang, S., Xu, J., 2015. Biosens. Bioelectron. 64, 147-153.

Liu, X., Wood, J. D, Chen, K. S., Cho, E., Hersam, M. C., 2015. J. Phys. Chem. Lett. 6, 773-778.

Ribeiro, H. B., Pimenta, M. A., De Matos, C, J, S., Moreira, R. L., Rodin, A. S., Zapata, J. D., De Souza, E. A. T., Castro Neto, A. H., 2015. ACS Nano 9, 4270-4276.

Saito, Y., Iwasa, Y., 2015. ACS Nano 9, 3192-3198.

Sreshtt, V., Padua, A. A. H., Blankschtein, D., 2015. ACS Nano 9, 8255-8268. 
Sun, Z., Xie, H., Tang, S., Yu, X. F., Guo, Z., Shao, J., Zhang, H., Huang, H., Wang, H., Chu, P. K., 2015. Angew. Chem. Int. Edit. 54, 11526-11530.

Wang, H., Yang, X., Shao, W., Chen, S., Xie, J., Zhang, X., Wang, J., Xie, Y.. 2015. J. Am. Chem. Soc. 137, 11376-11382.

Wang, X., Jones, A. M., Seyler, K. L, Vy, T., Jia, Y., Zhao, H., Wang, H., Yang, L., Xu, X., Xia, F., 2015. Nature Nanotech. 10, 517-521.

Woomer, A. H, Farnsworth, T. W., Hu, J., Wells, R. A., Donley, C. L., Warren, S. C., 2015. ACS Nano 9, 8869-8884.

Xiang, D., Han, C., Wu, J., Zhong, S., Liu, Y. Y., Lin, J. D., Zhang, X. A., Hu, W. P., Ozyilmaz, B., Neto, A. H. C., Wee, A. T. S, Chen, W., 2015. Nature Comm. 6, 6485.

Yasaei, P., Kumar, B., Foroozan, T., Wang, C., Asadi, M., Tuschel, D., Indacochea, J. E., Klie, R. F., Salehi-Khojin, A., 2015. Adv. Mater. 27, 1887-1892.

Zhang, J., Tu, L., Zhao, S., Liu, G., Wang, Y., Wang, Y., Yue, Z., 2015. Biosens. Bioelectron. 67, 296-302.

Zhang, X., Xie, H. M., Liu, Z. D., Tan, C. L., Luo, Z. M., Li, H., Lin, J. D., Sun, L. Q., Chen, W., Xu, Z. C., Xie, L. H., Huang, W., Zhang, H., 2015. Angew. Chem. Int. Edit. $54,3653-3657$. 